\title{
Estimativas de parâmetros genéticos para ferro, zinco, magnésio e fósforo em grãos de feijão
}

\author{
Juliana Sawada Buratto*, Vania Moda-Cirino
}

Instituto Agronômico do Paraná, Londrina,PR, Brasil

*Autor correspondente, e-mail: jusabu@gmail.com

\begin{abstract}
Resumo
Informações a respeito dos parâmetros genéticos são requeridas para o desenvolvimento de cultivares com maior valor nutricional. Neste sentido, este trabalho objetivou-se estimar os parâmetros genéticos: variâncias genéticas, coeficientes de herdabilidade no sentido restrito e amplo e efetuar a predição dos ganhos de seleção para os teores de Fe, Zn, Mg e P em grãos de feijão. O cruzamento foi feito entre as cultivares: FT Nobre x IPR Gralha e Diamante Negro x IPR Chopim. Os genitores $\left(P_{1}\right.$ e $\left.P_{2}\right)$ foram cruzados originando as gerações $F_{1}$ e $F_{2}$ e os retrocruzamentos $R C_{1}\left(P_{1} \times F_{1}\right)$ e RC $\left(P_{2} \times R C_{2}\right)$. Os teores de minerais Fe, $Z n$, Mg e $P$ nos grãos foram mensurados por digestão nítrico-perclórica e utilizando espectrometria de emissão atômica acoplada ao plasma (ICP-OES). As predições dos ganhos obtidos para o primeiro ciclo de seleção foram positivas indicando a viabilidade de incrementar os teores dos minerais Fe, Zn, Mg e P em grãos de feijão por meio de métodos de melhoramento clássicos. Com relação à predição dos ganhos obtidos no primeiro ciclo de seleção foi observado valores oscilando de 3,24\% a 15,48\% para $\mathrm{Mg}$ e $\mathrm{Fe}$, respectivamente. As estimativas de herdabilidade no sentido restrito variaram de $29,48 \%$ a $62,04 \%$ e as de herdabilidade no sentido amplo variaram de $45,14 \%$ a $76,36 \%$.
\end{abstract}

Palavras-chave: biofortificação, melhoramento genético, Phaseolus vulgaris L., valor nutricional

\section{Estimates of genetic parameters for iron, zinc, magnesium and phosphorus in common bean grains}

\begin{abstract}
Information about genetic parameters is required for the development of cultivars with higher nutritional value. In this sense, this study aimed to estimate the following genetic parameters: genetic variances, heritability coefficients in narrow and broad sense and predict the selection gain for the Fe, $\mathrm{Zn}, \mathrm{Mg}$ and $\mathrm{P}$ contents in common bean grains. The crosses were made between cultivars: FT Nobre $x$ IPR Gralha and Diamante Negro $x$ IPR Chopim. The genitors $\left(P_{1}\right.$ and $\left.P_{2}\right)$ were crossed resulting in $F_{1}$ and $F_{2}$ generations and backcrosses $B C_{1}\left(P_{1} \times F_{1}\right)$ and $B C_{2}\left(P_{2} \times R C_{2}\right)$. The mineral contents of $\mathrm{Fe}, \mathrm{Zn}, \mathrm{Mg}$ and $\mathrm{P}$ in grains were measured by nitric-perchloric digestion using atomic emission spectrometry coupled with plasma (ICP-OES). The predicted gains for the first cycle of selection were positive, indicating the viability of the increaseof the minerals Fe, $\mathrm{Zn}, \mathrm{Mg}$ and $\mathrm{P}$ levels on common bean grains using classical breeding methods. The prediction gains obtained in the first cycle of selection resulted in values ranging from $3.24 \%$ to $15.48 \%$ for $\mathrm{Mg}$ and Fe, respectively. Heritability estimates in narrow sense ranged from $29.48 \%$ to $62.04 \%$ and the broad range sense heritability ranged from $45.14 \%$ to $76.36 \%$.
\end{abstract}

Keywords: biofortification, genetic breeding, Phaseolus vulgaris L., nutritional value 


\section{Introdução}

Os micronutrientes são substâncias nos alimentos que são essenciais para a saúde humana e são requeridos em pequenas quantidades. Eles incluem todas as vitaminas conhecidas e minerais essenciais (Miller \& Welch, 2013). A desnutrição crônica e a deficiência de micronutrientes são problemas de saúde global que comprometem o bem estar humano (Welch \& Graham, 2004; Blair et al., 2009).

Diversas estratégias têm sido utilizadas para combater a desnutrição. A abordagem mais desejável é aumentar a diversidade de alimentos ingeridos, mas isto pode ser impraticável para as populações de baixa renda. Outra abordagem utilizada é a adição artificial de nutrientes por meio da suplementação ou fortificação de alimentos básicos como o sal e a farinha. A terceira estratégia é a "biofortificação", na qual as culturas agrícolas são modificadas ou melhoradas para acumular mais nutrientes (Perez-Massoti et al., 2013).

A "biofortificação" poder ser feita geneticamente utilizando duas maneiras. A primeira consiste no uso de técnicas de engenharia genética e a segunda estratégia engloba os métodos clássicos de melhoramento convencional. Um exemplo bem-sucedido do uso da engenharia genética é o arroz "Golden Rice" que apresenta altos teores de pro-vitamina A (Perez-Massoti et al., 2013).

$\mathrm{Na}$ cultura do feijão, a adoção de métodos convencionais de melhoramento é a estratégia mais utilizada para efetuar a "biofortificação" dos grãos. Este fato é justificável, pois existe ampla variabilidade genética para teores de diversos minerais no germoplasma da espécie Phaseolus vulgaris L. (Beebe et al., 2000; Mesquita et al., 2007; Gouveia et al., 2014) e relatos obtidos com ganhos de seleção para os minerais nos grãos (Jost et al., 2009a; 2009b; Rosa et al., 2010; Ribeiro et al., 2011).

Cultivares com maior valor nutricional é objetivo de programas de melhoramento de feijão e outras culturas alimentícias de importância econômica. Sendo assim, a adoção destas variedades é sustentável em longo prazo, pois alcança áreas rurais carentes, substituindo as cultivares menos nutritivas no sistema de produção e também contribui de forma indireta para a saúde da população (Nestel et al., 2006). Ressalta-se que o consumo de feijão deve ser estimulado, pois é vantajoso do ponto de vista nutricional. Na composição dos grãos são encontrados nutrientes importantes para o organismo humano, tais como: proteínas, fibras alimentares, carboidratos, vitaminas do complexo B, entre outros (Mesquita et al., 2007; Akond et al., 2011; Blair, 2013).

Programas de melhoramento que visamà obtenção de cultivares com alto valor nutricional agregado nos grãos requerem o conhecimento dos parâmetros genéticos, no qual estas informações servirão de subsídios na tomada de decisão para a escolha da metodologia mais adequada. Neste sentido, objetivou-se estimar os parâmetros genéticos: variâncias genéticas, coeficientes de herdabilidade do sentido restrito e amplo e efetuar a predição dos ganhos de seleção para os teores de Fe, Zn, Mg e P em grãos de feijão.

\section{Material e métodos}

Para estimar os parâmetros genéticos associados aos teores de Fe, Zn, Mg e P em grãos de feijão, efetuou-se a análise dos teores desses minerais nas gerações $P_{1}, P_{2^{\prime}} F_{1}, F_{2}, R C_{1}\left(P_{1}\right.$ $\left.x F_{1}\right)$ e $R C_{2}\left(P_{2} \times F_{1}\right)$ dos cruzamentos: FT Nobre $x$ IPR Gralha e Diamante Negro x IPR Chopim. Os genótipos FT Nobre e Diamante Negro foram utilizados como femininos e os genótipos IPR Gralha e IPR Chopim foram utilizados como masculinos. Todos os genitores são de origem mesoamericana, apresentam o tegumento de coloração preta e teores contrastantes para os minerais nos grãos (Tabela 1).

Os cruzamentos foram realizados manualmente em casa de vegetação onde inicialmente, foram feitas emasculações dos botões florais dos genitores femininos através da retirada das anteras com pinça, seguido pelas polinizações com pólen dos genitores masculinos. Para obtenção da geração $F_{1}$ e dos $R C_{1}\left(P_{1} \times F_{1}\right)$ e $R_{2}\left(P_{2} \times F_{1}\right)$ os genitores foram semeados em recipientes de plástico com a capacidade de 5 $\mathrm{L}$, contendo substrato composto por uma mistura na proporção de 1:3 de solo e areia. A água e nutrientes foram fornecidos de acordo com as 
necessidades exigidas pela cultura e resultado da análise de solo. O controle de pragas e doenças foi efetuado quando necessário, utilizando-se produtos registrados para a cultura do feijoeiro. Os recipientes foram dispostos em bancadas para facilitar o cruzamento dirigido na época de floração.

As sementes $F_{1}$ foram obtidas no período de março a junho de 2008, e posteriormente foram obtidas as sementes $F_{2}$ a partir da autofecundação natural das plantas $F_{1}$. Concomitantemente foram obtidas as gerações de retrocruzamentos $R C_{1}\left(P_{1} \times F_{1}\right)$ e $R C_{2}\left(P_{2} \times F_{1}\right)$. Em janeiro de 2009, correspondendo ao período da safra da seca, as gerações $P_{1}, P_{2^{\prime}} F_{1}, F_{2^{\prime}} R C_{1}$ $\left(P_{1} \times F_{1}\right)$ e $R C_{2}\left(P_{2} \times F_{1}\right)$, de cada cruzamento foram semeadas em dois locais, Ponta Grossa (altitude de 975 m; latitude 2505' Sul; longitude 5009'Oeste; solo classificado como Latossolo vermelho escuro álico) e Guarapuava (altitude de 1098 m; latitude 25²3' Sul; longitude 51 '27' Oeste; solo classificado como Cambissolo álico húmico). O delineamento experimental utilizado foi o inteiramente casualisado, utilizando 300 sementes da geração $F_{2}$ e 30 sementes nas demais gerações $\left(P_{1}, P_{2^{\prime}} F_{1}, R C_{1}\right.$ e $\left.R C_{2}\right)$, sendo cada planta considerada uma repetição. Os tratamentos foram dispostos em fileiras de 2,00 $\mathrm{m}$ de comprimento, espaçadas $0,50 \mathrm{~m}$, com uma densidade de 15 plantas por metro linear. A adubação de base foi efetuada de acordo com análise química do solo e efetuou-se uma adubação nitrogenada em cobertura com 100 $\mathrm{kg}$. $\mathrm{ha}^{-1}$ de ureia no estádio de desenvolvimento V3. O controle de pragas, doenças e plantas invasoras foi efetuado com produtos registrados para a cultura.

As plantas de cada geração foram colhidas individualmente, e todas as vagens de cada uma das plantas foram colocadas em sacos de papel kraft com capacidade para $5 \mathrm{~kg}$, e posteriormente debulhadas. Uma amostra aleatória de grãos colhidos de cada planta, contendo no mínimo 10 gramas, foi enviada ao Laboratório de Análises Químicas de Tecido Vegetal e Solos localizado no IAPAR de LondrinaPR.

No laboratório as amostras de grãos de feijão foram inicialmente lavadas em água corrente de torneira, e em seguida foram lavadas com água destilada para retirar qualquer resíduo de solo ou outro material que possa vir aderido aos grãos. Após a lavagem, as amostras foram secas em estufa de ventilação constante a 65 ${ }^{\circ} \mathrm{C}$. As amostras foram moídas até a obtenção de partículas inferiores a 1,00 mm de diâmetro (Moinho triturador para análises da marca IKA, modelo Al 1 Basic). Em um tubo de digestão, foi colocado 0,4 g da farinha de feijão cru, a qual foi digerida em $4 \mathrm{ml}$ de solução nitroperclórica $\left(\mathrm{HNO}_{3}+\mathrm{HClO}_{4}\right)$, na proporção volumétrica de 3:1, conforme a metodologia descrito por Miyazawa et al. (1999). A leitura dos teores de minerais foi realizada por espectrometria de emissão atômica acoplada ao plasma (ICP-OES) (marca Thermo Jarrell - Ash, modelo ICAP 61E).

O teste t a $5 \%$ de probabilidade foi usado para testar os contrastes $P_{1}$ vs $P_{2^{\prime}} P_{1} v s F_{1}$ e $P_{2} v s F_{1}$ sendo efetuado com o auxílio do Programa $R$ ( $R$ Core Development Team, 2010). As estimativas dos parâmetros genéticos foram obtidas a partir das variâncias das gerações avaliadas em que $\sigma_{P 1}^{2}, \sigma_{P 2}^{2}, \sigma_{F 1}^{2}, \sigma_{F 2}^{2}, \sigma_{R 1}^{2}$ e $\sigma_{R 2}^{2}$ correspondem respectivamente à variância dos genitores $P_{1}$ e $P_{2}$ e das gerações $F_{1}, F_{2}, R C_{1}$ e $R C_{2}$. Para $a$ estimativa destes parâmetros foi considerada a geração do embrião (cotilédones e eixo embrionário) da semente. As herdabilidades no sentido amplo $\left(h_{a}^{2}=\sigma_{G}^{2} / \sigma_{F}^{2}\right)$ e a herdabilidade no sentido restrito $\left(h_{r}^{2}=\sigma_{A}^{2} / \sigma_{F}^{2}\right)$ foram estimadas considerando as seguintes expressões: variância aditiva $\left(\sigma_{A}^{2}=2 \sigma_{F 2}^{2}-\left(\sigma_{R C 1}^{2}+\sigma_{R C 2}^{2}\right)\right.$, a variância fenotípica $\left(\sigma_{F}^{2}=\sigma_{F 2}^{2}\right)$ e a variância ambiental em $\mathrm{F}_{2}\left(\sigma_{E}^{2}=1 / 4\left(\sigma_{P 1}^{2}+\sigma_{P 2}^{2}+\sigma_{F 1}^{2}\right)\right.$ conforme descrito por Cruz \& Regazzi (1994) e utilizado por Rosa et al., (2010). A predição do ganho de seleção $(\Delta G)$ foi estimada usando as seguintes fórmulas: $\left(\Delta G=D S \cdot h_{a}^{2}\right)$ e ( $\Delta G(\%)=(\Delta G \times 100) /\left(\bar{F}_{2}\right)$ em que o DS é o diferencial de seleção, que é expresso por $D S=\bar{X}_{S}-\bar{X}_{o}$, onde $\bar{X}_{S}$ corresponde à média das plantas $F_{2}$ selecionadas e $\bar{X}_{o}$ corresponde à média das populações originais das plantas com sementes $F_{2}$. Para o cálculo da predição do ganho por seleção foram considerados $20 \%$ dos indivíduos $F_{2}$ que apresentaram os maiores teores de Fe, Zn, Mg e P. As análises estatísticas foram efetuadas com o auxílio do programa Genes 
(Cruz, 2013).

\section{Resultados e discussão}

Os contrastes entre os genitores $P_{1}$ vs $\mathrm{P}_{2}$ foram significativos pelo teste $t$ a 1 ou $5 \%$ de probabilidade para a maioria dos teores de minerais avaliados (Tabela 1), demonstrando a existência de divergência entre os genitores utilizados. Deste modo, a ocorrência de variabilidade genética dentro da espécie torna viável incrementar os teores de micro e macro nutrientes minerais por meio de métodos de melhoramento convencionais (Pfeiffer \& Mcclafferty, 2007).

Para o teor de Fe nos grãos, com o cultivo em Guarapuava, o contraste entre $P_{1}$ vs $F_{1}$ não foi significativo, esse fato indica que o teor médio de ferro do $P_{1}$ utilizado como genitor feminino não diferiu estatisticamente pelo teste $\dagger$ em relação à média do híbrido $F_{1}$ (Tabela 1). No entanto, quando foi realizado o cultivo em Ponta Grossa, os contrastes entre $P_{1}$ vs $F_{1}$ foram significativos $(p<0,001)$, diferindo dos resultados apresentados em Guarapuava. Logo, supõe-se que esses resultados podem ter sido influenciados pelo efeito ambiental. Os estudos realizados por Moraghan et al. (2002) e Pereira et al. (2014) comprovam que o ambiente de cultivo (condições climáticas e edáficas) afeta a acumulação do ferro nos grãos de linhagens e cultivares de feijão. Tratando-se especificamente de condições edáficas, Moraghan et al. (2002) verificaram que sementes oriundas de plantas cultivadas em solos ácidos $(\mathrm{pH}=6,0)$ apresentavam teores de $\mathrm{Fe} 25 \%$ superiores em relação àquelas cultivadas em solos alcalinos $(\mathrm{pH}=8,2)$, isso deve-se ao fato que a absorção de ferro pela planta em solos bem aerados é dependente da solubilidade do ferro no solo, a qual é afetada pelo $\mathrm{pH}$.

Para o teor de Zn, os contrastes $P_{1}$ vs $F_{1}$ (FT Nobre x IPR Gralha em Ponta Grossa; Diamante Negro x IPR Chopim em Guarapuava e Ponta Grossa), indicam que as médias testadas no contraste não diferem estatisticamente (Tabela 1). Este resultado sugere que a constituição genética da cultivar utilizada como genitor feminino pode influenciar o fenótipo dos seus descendentes, indicando a possível ocorrência de efeito materno. As informações encontradas na literatura são divergentes a respeito da atuação do efeito materno para zinco em grãos de feijão. Não foi detectado efeito materno nos genótipos estudados por Moraghan et al. (2002), diferindo dos resultados apresentados por Silva et al., (2013).

O teor de $\mathrm{Mg}$ em grãos de feijão apresenta variabilidade genética, este fato foi observado somente entre as cultivares FT Nobre e IPR Gralha. Os contrastes $P_{1}$ vs $P_{2^{\prime}} P_{1} v s F_{1}$ e $P_{2}$ vs $F_{1}$ foram significativos pelo teste $\dagger$ a $1 \%$ de probabilidade no cruzamento FT Nobre x IPR Gralha para o teor de Mg (Tabela 1), indicando diferença entre as médias dos genitores e entre os genitores e o $F_{1}$. No cruzamento entre Diamante Negro x IPR Chopim nenhum contraste testado pelo teste $\dagger$ foi significativo (Tabela 1). Para o teor de $P$, os contrastes $P_{1} v s F_{1}$ e $P_{2} v s F_{1}$ foram significativos no cruzamento FT Nobre $x$ IPR Gralha, portanto os teores médios de $\mathrm{P}$ nos grãos dos genitores diferem do híbrido $F_{1}$, enquanto que no cruzamento Diamante Negro $x$ IPR Chopim o contraste entre $P_{1}$ vs $F_{1}$ não foi significativo (Tabela 1). Para os teores de $\mathrm{Mg}$ e $P$ em grãos de feijão, supõe-se a ausência de efeito materno, pois maior fração deste mineral encontra-se no embrião das sementes de feijão (Moraghan et al., 2006).

A presença de variabilidade genética é um pré-requisito no melhoramento, este fato pode ser verificado pela decomposição da variância fenotípica, em que foi observado a predominância dos efeitos genéticos em relação aos efeitos ambientais nos cruzamentos FT Nobre x IPR Gralha e Diamante Negro x IPR Chopim para os teores de Fe, Zn, Mg e P nos grãos (Tabela 2). Nas estimativas das variâncias genéticas observam-se a predominância de efeitos genéticos aditivos (Tabela 2). A variância genética aditiva constitui-se em um indicativo da facilidade de identificação de genótipos geneticamente superiores, que poderão proporcionar ganhos vantajosos em virtude de sua seleção (Cruz \& Regazzi, 1994). Este resultado indica que a estratégia mais adequada para um programa de melhoramento seria a obtenção de linhagens ou o uso da seleção recorrente (Blair et al., 2009). Considerando que o feijoeiro 
é uma espécie autógama este processo torna-

se viável.

Tabela 1. Média dos teores dos minerais Fe, Zn, Mg e P mensuradas em genótipos de feijão em distintas gerações: $P_{1}, P_{2}, F_{1}, F_{2}, R C_{1}\left(P_{1} \times F_{1}\right)$ e RC $\left(P_{2 x} F_{1}\right)$ obtidos a partir dos cruzamentos: FT Nobre $x$ IPR Gralha e Diamante Negro X IPR Chopim, cultivados em Guarapuava (GUA) e Ponta Grossa (PG) no estado do Paraná na safra seca 2009

\begin{tabular}{|c|c|c|c|c|c|c|c|c|c|c|c|}
\hline \multirow{2}{*}{ Mineral } & \multirow{2}{*}{$\begin{array}{l}\text { Cruzamento } \\
\left(\mathrm{P}_{1} \times \mathrm{P}_{2}\right)\end{array}$} & \multicolumn{7}{|c|}{ Genitores e gerações } & \multicolumn{3}{|c|}{ Contrastes } \\
\hline & & Locall $^{/ 1}$ & $P_{1}$ & $\mathrm{P}_{2}$ & $F_{1}$ & $\mathrm{~F}_{2}$ & $\mathrm{RC}_{1}$ & $\mathrm{RC}_{2}$ & $\mathrm{P}_{1} \vee s \mathrm{P}_{2}$ & $\mathrm{P}_{1} \vee s \mathrm{~F}_{1}$ & $\mathrm{P}_{2} v_{s} \mathrm{~F}_{1}$ \\
\hline \multirow{4}{*}{$\begin{array}{c}\mathrm{Fe} \\
\left(\mathrm{mg} \cdot \mathrm{kg}^{-1}\right)\end{array}$} & FT Nobre x IPR Gralha & GUA & 84,90 & 71,00 & 89,20 & 86,00 & 77,60 & 70,20 & ** & ns & ** \\
\hline & FT Nobre x IPR Gralha & PG & 83,35 & 76,34 & 73,43 & 80,31 & 79,47 & 85,34 & $*$ & ** & ns \\
\hline & D. Negro x IPR Chopim & GUA & 72,20 & 83,40 & 74,70 & 75,40 & 66,40 & 75,80 & ** & ns & ** \\
\hline & D. Negro x IPR Chopim & $P G$ & 70,40 & 74,09 & 78,82 & 73,71 & 71,88 & 71,76 & $* *$ & ** & $*$ \\
\hline \multirow{4}{*}{$\begin{array}{c}\mathrm{Zn} \\
\left(\mathrm{mg}^{-\mathrm{kg}^{-1}}\right)\end{array}$} & FT Nobre x IPR Gralha & GUA & 26,96 & 32,60 & 32,22 & 28,34 & 35,92 & 29,70 & ** & ** & ns \\
\hline & FT Nobre x IPR Gralha & $P G$ & 38,73 & 35,70 & 40,90 & 41,78 & 40,42 & 44,33 & $*$ & ns & ** \\
\hline & D. Negro x IPR Chopim & GUA & 32,42 & 35,45 & 30,52 & 31,19 & 25,21 & 31,95 & * & ns & * \\
\hline & D. Negro x IPR Chopim & $P G$ & 37,60 & 36,73 & 39,08 & 38,41 & 38,13 & 35,90 & ns & ns & ns \\
\hline \multirow{2}{*}{$\begin{array}{c}M g \\
\left(g . \mathrm{kg}^{-1}\right)\end{array}$} & FT Nobre x IPR Gralha & GUA & 1,936 & 2,030 & 2,169 & 2,104 & 2,046 & 2,102 & ** & ** & ** \\
\hline & D. Negro x IPR Chopim & GUA & 2,036 & 2,019 & 2,067 & 2,022 & 2,006 & 2,023 & ns & ns & ns \\
\hline \multirow{2}{*}{$\begin{array}{c}\mathrm{P} \\
\left(\mathrm{g} \cdot \mathrm{kg}^{-1}\right)\end{array}$} & FT Nobre x IPR Gralha & GUA & 7,579 & 5,144 & 7,057 & 5,153 & 5,705 & 6,267 & ** & ** & ** \\
\hline & D. Negro x IPR Chopim & GUA & 5,545 & 5,993 & 5,697 & 5,906 & 5,903 & 6,090 & ** & ns & ** \\
\hline
\end{tabular}

De maneira geral, os teores de minerais

avaliados apresentaram estimativas de herdabilidade moderadas a altas (Tabela 2). As estimativas dos coeficientes de herdabilidade no sentido amplo $\left(h_{a}^{2}\right)$ variaram de $45,14 \%$ a $76,36 \%$. As estimativas dos coeficientes de herdabilidade

no sentido restrito $\left(h_{r}^{2}\right)$ variaram de $29,48 \%$ a $62,04 \%$ (Tabela 2). Considerando a magnitude das estimativas de herdabilidade no sentido restrito $\left(h_{r}^{2}\right)$, é possível obter ganhos efetuando a seleção em gerações precoces.

Tabela 2. Estimativas de parâmetros genéticos dos teores dos minerais $\mathrm{Fe}, \mathrm{Zn}, \mathrm{Mg}$ e $\mathrm{P}$ em grãos de de feijão proveniente dos cruzamento: FT Nobre x IPR Gralha e Diamante Negro x IPR Chopim cultivados em Guarapuava (GUA) e Ponta Grossa (PG) no estado do Paraná na safra seca 2009

\begin{tabular}{|c|c|c|c|c|c|c|c|c|c|}
\hline Mineral & Cruzamento & Local & $\mathrm{VF}^{\prime / 1}$ & $\mathrm{VE}^{/ 2}$ & $\mathrm{VG}^{/ 3}$ & $\mathrm{VA}^{/ 4}$ & $\mathrm{VD}^{/ 5}$ & $\mathrm{~h}^{2}{ }^{16}$ & $\mathrm{~h}_{\mathrm{r}}^{2 / 7}$ \\
\hline \multirow{4}{*}{ 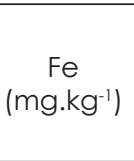 } & FT Nobre x IPR Gralha & GUA & 262,100 & 82,420 & 179,760 & 125,150 & 54,600 & 68,56 & 47,73 \\
\hline & FT Nobre x IPR Gralha & $P G$ & 190,870 & 79,260 & 111,610 & 87,260 & 24,350 & 58,47 & 45,71 \\
\hline & D. Negro x IPR Chopim & GUA & 150,680 & 36,800 & 113,870 & 93,490 & 20,380 & 75,57 & 62,04 \\
\hline & D. Negro x IPR Chopim & PG & 104,010 & 41,560 & 62,440 & 61,650 & 0,7980 & 60,04 & 59,27 \\
\hline \multirow{4}{*}{$\begin{array}{c}\mathrm{Zn} \\
\left(\mathrm{mg}^{-\mathrm{kg}^{-1}}\right)\end{array}$} & FT Nobre x IPR Gralha & GUA & 21,607 & 9,246 & 12,360 & 9,686 & 2,675 & 57,20 & 44,82 \\
\hline & FT Nobre x IPR Gralha & $P G$ & 47,406 & 24,763 & 22,643 & 13,979 & 8,663 & 47,76 & 29,48 \\
\hline & D. Negro x IPR Chopim & GUA & 49,074 & 16,838 & 32,236 & 28,205 & 4,030 & 65,68 & 57,47 \\
\hline & D. Negro x IPR Chopim & $P G$ & 36,255 & 19,889 & 16,365 & 15,402 & 0,963 & 45,14 & 42,48 \\
\hline \multirow{2}{*}{$\begin{array}{c}\mathrm{Mg} \\
\left(\mathrm{g} \cdot \mathrm{kg}^{-1}\right)\end{array}$} & FT Nobre x IPR Gralha & GUA & 0,039 & 0,013 & 0,026 & 0,023 & 0,0026 & 66,64 & 59,81 \\
\hline & D. Negro x IPR Chopim & GUA & 0,024 & 0,122 & 0,011 & 0,007 & 0,0047 & 49,26 & 29,66 \\
\hline \multirow{2}{*}{$\begin{array}{c}P \\
\left(g . \mathrm{kg}^{-1}\right)\end{array}$} & FT Nobre x IPR Gralha & GUA & 0,462 & 0,109 & 0,353 & 0,244 & 0,108 & 76,36 & 52,87 \\
\hline & D. Negro x IPR Chopim & GUA & 0,498 & 0,122 & 0,376 & 0,302 & 0,074 & 75,47 & 60,63 \\
\hline
\end{tabular}

seleção foram considerados $20 \%$ dos indivíduos $\mathrm{F}_{2}$ que apresentaram os maiores teores de $\mathrm{Fe}, \mathrm{Zn}$, Mg e P.

As estimativas das predições dos ganhos por seleção $(\Delta G)$ variaram em função do mineral avaliado, população estudada e das condições do ambiente as quais as plantas foram submetidas durante o cultivo (Tabela 3). Para o cálculo da predição do ganho por
$\mathrm{Na}$ avaliação feita no banco de germoplasma de feijão do CIAT, foi constatado que é possível aumentar em até $80 \%$ o conteúdo de Fe nas sementes e 50\% o teor de Zn (Beebe et al., 2000). As predições dos ganhos com a seleção obtidas neste trabalho para estes 
minerais foram mais modestas chegando a $14,25 \%$ e $37,09 \%$ para Fe e $Z n$, respectivamente (Tabela 3).

O teor de Fe no cruzamento FT Nobre x IPR Gralha apresentou a maior média predita $\left(X_{1} s=98,24 \mathrm{mg} \cdot \mathrm{kg}^{-1}\right)$ após o primeiro ciclo de seleção (Tabela 3). No entanto, um ponto a ser levado em consideração é a questão da biodisponibilidade dos nutrientes minerais para o organismo humano, uma vez que a presença de fatores antinutricionais, como os fitatos e os compostos fenólicos presentes nos grãos, reduz a biodisponibilidade desses minerais para 0 organismo humano (Frossard et al., 2000). Porém, no estudo realizado por Tako et al. (2011) concluise que feijões biofortificados com altos teores de ferro nos grãos constituem um instrumento promissor para aumentar a biodisponibilidade de ferro para a população humana, pois os efeitos inibitórios dos polifenóis na absorção de ferro são neutralizados pelo incremento da concentração de ferro nos grãos.

A necessidade diária dos minerais $\mathrm{Fe} e$ Zn para um indivíduo adulto, na faixa etária de 25 a 50 anos é de 10 a $15 \mathrm{mg}$ de ferro e 12 a $15 \mathrm{mg}$ de zinco (Welch \& Graham, 2004). Apesar da pequena quantidade requerida, deficiência dos minerais Fe e Zn afligem milhares de pessoas, portanto um suprimento adequado destes elementos na dieta ajudam a prevenir a anemia ferropriva e a deficiência de zinco (Blair et al., 2009).

Cultivares com maiores teores de $\mathrm{Zn}$ nos grãos apresentam a vantagem de proporcionar maior qualidade à dieta humana e também para a sustentabilidade dos cultivos em áreas agrícolas deficientes em zinco. No cruzamento FT Nobre x IPR Gralha o ganho de seleção estimado em Guarapuava foi de 11,62\% para zinco. O ganho de seleção para o mineral zinco no cruzamento Diamante Negro x IPR Chopim foi de $18,96 \%$ quando realizada em Guarapuava, e de 10,52 \% em Ponta Grossa. Rosa et al. (2010) relatou ganho por seleção de até 12,22\% para teor de Zn em grãos de feijão.

Em relação ao $P$, os jovens e as mulheres grávidas ou em lactação são os grupos que apresentam os maiores requerimentos desse mineral na dieta (Frossard et al., 2000). O cruzamento mais promissor para este mineral foi o Diamante Negro x IPR Chopim com maior média predita no primeiro ciclo de seleção. Nos grãos de feijão, grande parte do P encontrado está na forma de fitatos, e esses compostos químicos são conhecidos por suas propriedades antinutricionais, que apresentam forte interação com ferro e zinco tornando-os não biodisponíveis para o organismo humano. Por outro lado, os fitatos são a principal forma de armazenamento de P na semente (Frossard et al., 2000).

O cruzamento FT Nobre x IPR Gralha demonstrou ser mais promissor por apresentar maior média predita após o primeiro ciclo de seleção para o teor de Mg (Tabela 3). A importância do $\mathrm{Mg}$ no organismo humano reside na sua participação em vários sistemas enzimáticos, manutenção do potencial elétrico dos nervos e membranas e do uso na liberação de energia para a contração muscular e para o metabolismo de Ca e P (Frossard et al., 2000).

Tabela 3. Estimativas dos parâmetros genéticos associados aos ganhos de seleção para os teores de Fe, $\mathrm{Zn}, \mathrm{Mg}$ e P nos grãos de feijão proveniente dos cruzamentos: FT Nobre x IPR Gralha e Diamante Negro x IPR Chopim cultivados em Guarapuava (GUA) e Ponta Grossa (PG) no estado do Paraná, safra seca 2009

\begin{tabular}{|c|c|c|c|c|c|c|c|c|c|}
\hline Mineral & Cruzamento & Local & $\mathrm{XO}^{/ 1}$ & $\mathrm{XS}^{1 / 2}$ & $\mathrm{NIS}^{/ 3}$ & $\mathrm{DS}^{/ 4}$ & $\Delta \mathrm{G}^{15}$ & $\Delta \mathrm{G}(\%)^{1 / 6}$ & $\mathrm{X}, \mathrm{S}^{17}$ \\
\hline \multirow{4}{*}{$\begin{array}{c}\mathrm{Fe} \\
\left(\mathrm{mg}^{\left.-\mathrm{kg}^{-1}\right)}\right.\end{array}$} & FT Nobre x IPR Gralha & GUA & 85,99 & 111,66 & 46 & 25,67 & 12,25 & 14,25 & 98,24 \\
\hline & FT Nobre x IPR Gralha & PG & 80,31 & 100,85 & 57 & 20,53 & 9,38 & 11,68 & 89,70 \\
\hline & D. Negro x IPR Chopim & GUA & 75,36 & 94,17 & 58 & 18,80 & 11,67 & 15,48 & 87,03 \\
\hline & D. Negro x IPR Chopim & PG & 73,71 & 89,59 & 51 & 15,88 & 9,41 & 12,77 & 83,13 \\
\hline \multirow{4}{*}{$\begin{array}{c}\mathrm{Zn} \\
\left(\mathrm{mg} \cdot \mathrm{kg}^{-1}\right)\end{array}$} & FT Nobre x IPR Gralha & GUA & 28,34 & 35,69 & 46 & 7,35 & 3,29 & 11,62 & 31,63 \\
\hline & FT Nobre x IPR Gralha & PG & 41,78 & 52,15 & 57 & 10,36 & 3,05 & 7,31 & 44,84 \\
\hline & D. Negro x IPR Chopim & GUA & 31,19 & 41,47 & 59 & 10,28 & 5,91 & 18,96 & 37,09 \\
\hline & D. Negro x IPR Chopim & PG & 38,41 & 47,93 & 51 & 9,51 & 4,04 & 10,52 & 41,45 \\
\hline \multirow{2}{*}{$\begin{array}{c}M g \\
\left(g \cdot \mathrm{kg}^{-1}\right)\end{array}$} & FT Nobre x IPR Gralha & GUA & 2,10 & 2,41 & 46 & 0,30 & 0,18 & 8,59 & 2,29 \\
\hline & D. Negro x IPR Chopim & GUA & 2,02 & 2,25 & 59 & 0,22 & 0,07 & 3,24 & 2,09 \\
\hline \multirow{2}{*}{$\begin{array}{c}P \\
\left(g \cdot \mathrm{kg}^{-1}\right)\end{array}$} & FT Nobre x IPR Gralha & GUA & 5,15 & 6,21 & 46 & 1,06 & 0,56 & 10,88 & 5,71 \\
\hline & D. Negro x IPR Chopim & GUA & 5,91 & 6,98 & 59 & 1,07 & 0,65 & 10,97 & 6,55 \\
\hline
\end{tabular}


O valor nutricional apresentado pelos grãos de feijão é relevante, no entanto para que os agricultores adotem estas cultivares é necessário que as mesmas também apresentem bom potencial agronômico (rendimento de grãos e tolerância a patógenos) e alta aceitabilidade pelo consumidor para que 0 sucesso na comercialização seja garantido. De acordo com os resultados obtidos neste trabalho, é viável incrementar os teores de minerais $\mathrm{Fe}, \mathrm{Zn}, \mathrm{P}$ e $\mathrm{Mg}$ nos grãos utilizando métodos de melhoramento convencionais. Este fato possibilita o desenvolvimento de linhagens com alto valor nutritivo agregado nos grãos. Estas futuras linhagens poderão ser recomendadas para cultivo se tiver mérito agronômico ou ser utilizadas como genitoras em programas de melhoramento.

\section{Conclusões}

As variâncias genéticas predominaram em relação à variação ambiental para os teores dos minerais Fe, $\mathrm{Zn}, \mathrm{Mg}$ e P. As estimativas de herdabilidade no sentido restrito variaram de $29,48 \%$ a $59,81 \%$, enquanto que a herdabilidade no sentido amplo variaram de $45,14 \%$ a $76,36 \%$. Os valores preditos para os ganhos de seleção nos minerais podem alterar em função do local de cultivo e dos genitores utilizados no cruzamento.

\section{Referências}

Akond, A.S.M.G.M., Crawford, H., Berthold, J., Talukder, Z.I., Hossain, K. 2011. Minerals (Zn, $\mathrm{Fe}, \mathrm{Ca}$ and $\mathrm{Mg}$ ) and antinutrient (phytic acid) constituents in common beans. American Journal of Food Technology 6: 235-243.

Beebe, S., Gonzalez, A.V., Rengifo, J. 2000. Research on trace minerals in the common bean. Food and Nutrition Bulletin 21: 387-391.

Blair, M.W., Astudillo, C., Grusak, M.A., Graham, R., Beebe, S.E. 2009. Inheritance of seed iron and zinc concentration in common bean (Phaseolus vulgaris L.). Molecular Breeding 23: 197-207.

Blair, M.W. 2013. Mineral biofortification strategies for food staples: The example of common bean. Journal of Agricultural and Food Chemistry 61: 8287-8294.

Cruz C.D., Regazzi, A.J. 1994. Modelos biométricos aplicados ao melhoramento genético. UFV, Viçosa, Brasil. 309p.
Cruz, C.D. 2013. GENES - a software package for analysis in experimental statistics and quantitative genetics. Acta Scientiarum Agronomy 35: 271276.

Frossard, E., Bucher, M., Mächler, F., Mozafar, A., Hurrell, R. 2000. Potential for increasing the content and bioavailability of $\mathrm{Fe}, \mathrm{Zn}$ and $\mathrm{Ca}$ in plants for human nutrition. Journal of the Science of Food and Agriculture 80: 861-879.

Gouveia, C.S.S., Freitas, G., Brito, J.H., Slaski, J.J., Carvalho, M.A.A.P. 2014. Nutritional and mineral variability in 52 accessions of common bean varieties (Phaseolus vulgaris L.) from madeira island. Agricultural Sciences 5:317-329.

Jost, E., Riberio, N.D., Maziero, S. M., Cerutti, T., Rosa, D.P. 2009a. Efeitos gênicos do teor de cálcio em grãos de feijão. Ciência Rural 39: 31 37.

Jost, E., Riberio, N.D., Cerutti, T., Poersch, N.L., Maziero, S.M. 2009b. Potencial do aumento do teor de ferro em grãos de feijão por melhoramento genético. Bragantia 68: 35-42.

Mesquita, F.R., Correa, A. D., Abreu, C.M.P, Lima, R.A.Z., Abreu, A.F.B. 2007. Linhagens de feijão (Phaseolus vulgaris L.): composição química e digestibilidade protéica. Ciência e Agrotecnologia 31: 1114-1121.

Miller, D.D., Welch, R. M. 2013. Food system strategies for preventing micronutrient malnutrition. Food Policy 43: 115-128.

Miyazawa, M., Pavan, M.A., Muraoka, T., Carmo, C.A.F.S., Mello, W.J. 1999. Análises químicas de tecido vegetal. In: Silva, F. C. (Org.) Manual de análises químicas de solos, plantas e fertilizantes. Brasília: Embrapa Solos/ Embrapa Informática Agropecuária/ Embrapa Comunicação para a transferência de Tecnologia, Brasília, Brasil, p. 171-223.

Moraghan, J.T., Padilla, J., Ełchevers, J.D., Grafton, K., Acosta-Gallegos, J.A. 2002. Iron accumulation in seed of common bean. Plant and Soil 246: 175-183.

Moraghan, J.T., Ełchevers, J.D., Padilla, J. 2006. Contrasting accumulations of calcium and magnesium in seed coat and embryos of common bean and soybean. Food Chemistry 95: 554- 561.

Nestel, P., Bouis, H.E., Meenakshi, J.V., Pfeiffer, W. 2006. Biofortification of staple food crops. Journal of Nutrition 136:1064-1067.

Pereira, H.S., Del Peloso, M.J., Bassinello, P.Z., Guimarães, C.M., Melo, L.C., Faria, L.C. 2014. Genetic variability for iron and zinc content in common bean lines and interaction with water 
availability. Genetic and Molecular Research 13: 6773:6785.

Perez-Massot, E., Banakar, R., Gomez-Galera, S., Zorrilla-Lopez, U., Sanahuja, G., Arjó, G., Miralpeix, B., Vamvaka, E., Farre, G., Rivera, S.M., Dashevskaya, S., Berman, J., Sabalza, M., Yuan, D., Bai, C., Bassie, L., Twyman, R.M., Capell, T., Christou, P., Zhu, C. 2013. The contribution of transgenic plants to better health throughimproved nutrition: opportunities and constraints. Genes \& Nutrition 8: 29-41.

Pfeiffer, W.H., Mcclafferty, B. 2007. Harvest plus: breeding crops for better nutrition. Crop Science 47: 88-105.

R Development Core Team. R: a language and environment for statistical computing. 2009. http://www.r-project.org/ <Acesso em: 1 nov. 2009>

Ribeiro, N.D., Rosa, S.S., Jost, E., Rosa, D.P., Poerch, N.L., Maziero, S.M. 2011. Genetics of phosphorus content in common bean seeds. Crop Breeding and Applied Biotechnology 11:250-256.

Rosa, S.S., Ribeiro, N.D., Jost, E., Reiniger, L.R.S., Rosa, D.P., Cerutti, T., Possobom, M.T.D.F. 2010. Potential for increasing the zinc content in common bean using genetic improvement. Euphytica 175: 207-213.

Silva, C.A., Abreu, A.F.B., Ramalho, M.A.P. 2013. Genetic control of zinc and iron concentration in common bean seeds. African Journal of Agricultural Research 8(11): 1001-1008.

Tako, E.; Blair, M.W.; Glahn, R.P. 2011. Biofortified red mottled beans (Phaseolus vulgaris L.) in a maize and bean diet provide more bioavailable iron than standard red mottled beans: Studies in poultry (Gallus gallus) and in vitro digestion/ Caco-2 model. Nutrition Journal 10: 1-10.

Welch, R. M., Graham, R. D. 2004. Breeding for micronutrients in staple food crops from a human nutrition perspective. Journal of Experimental Botany 55: 353-364. 